\title{
Hot-Wire Measurements of the Influence of Surface Steps on Transition in Favorable Pressure Gradient Boundary Layers
}

\author{
S. Gerashchenko ${ }^{1}$ and B. J. McKeon ${ }^{2}$ \\ California Institute of Technology, Pasadena, CA 91125 \\ R. V. Westphal ${ }^{3}$ \\ California Polytechnic State University, San Luis Obispo, CA 93407 \\ A. M. Bender ${ }^{4}$ and A. Drake ${ }^{5}$ \\ Northrop Grumman Corporation, El Segundo, CA 90245
}

\begin{abstract}
An examination of the effects of surface step excrescences on boundary layer transition was performed, using a unique experimental facility. The objective of the work was to characterize the variation of transition Reynolds numbers with measurable step size and boundary layer parameters, with the specific goal of specifying new tolerance criteria for laminar flow airfoils, alongside a fundamental investigation of boundary layer transition mechanisms. This paper focuses on interpretation of hot-wire measurements, including supporting stability calculations, undertaken as part of the study. The results for both forward and aft-facing steps indicated a substantial stabilizing effect of favorable pressure gradient on excrescence-induced boundary layer transition. These findings suggest that manufacturing tolerances for laminar flow aircraft could be loosened in areas where even mild favorable pressure gradients exist.
\end{abstract}

\section{Nomenclature}

$\mathrm{X}$

S

k

$\mathrm{A}(\mathrm{s})$

$\mathrm{N}$

K

$\mathrm{C}_{\mathrm{f}}$

$\mathrm{Cp}$

$\mathrm{H}$

$\beta$

S*

$\delta *_{\mathrm{k}}$

$\mathrm{Re}$

U

$\mathrm{u}_{\mathrm{k}}$

$\mathrm{Re}_{\mathrm{k}}$

$\mathrm{Re}_{\mathrm{xk}}$

$\mathrm{Re}_{\mathrm{tr}}$

v

$\rho$

chord-wise distance

stream-wise distance measured along the surface

excrescence height

instability amplitude at stream-wise location $\mathrm{s}$

exponential amplification factor ( $\mathrm{N}$-factor)

acceleration parameter

skin friction coefficient

coefficient of pressure

shape factor

pressure parameter for Falkner-Skan flows

displacement thickness

displacement thickness at step location

Reynolds number based on free stream velocity

velocity at boundary layer edge

velocity in boundary layer profile at height $\mathrm{k}$

excrescence height Reynolds number

excrescence location Reynolds number

transition Reynolds number

kinematic viscosity

density

\footnotetext{
${ }^{1}$ Postdoctoral scholar. Current affiliation: Cornell University.

${ }^{2}$ Assistant Professor of Aeronautics, Graduate Aerospace Laboratories (GALCIT). AIAA Member.

${ }^{3}$ Professor, Mechanical Engineering. AIAA Senior Member.

${ }_{5}^{4}$ Aerodynamics Engineer, Aerodynamic Design and Analysis

${ }^{5}$ Manager, Flight Analysis. AIAA Senior Member.
} 


\section{I.A. Motivation and goals}

\section{Introduction}

Drag reduction on aircraft is given substantial attention in the design process for new long-range or long-endurance Intelligence, Surveillance, and Reconnaissance (ISR) aircraft, where efficient flight is of a high priority. For many aircraft designs skin friction drag is a dominant source of overall drag. Designing aircraft surfaces (in particular, wings) to have lower skin friction drag through the control of the boundary layer transition location has been pursued for many decades. The resulting drag reductions possible with such a "laminar flow aircraft" are often projected to be substantial.

To prevent premature transition on laminar flow aircraft, there is a need to understand excrescence effects and to develop appropriate surface tolerance requirements. Existing literature on laminar flow does not provide sufficient criteria on manufacturing tolerances for a surface in a pressure gradient. Building on the experimental and computational work performed as part of the Manufacturing Tolerances for Laminar Flow (MEATLOAF) project ${ }^{1,2}$, this study (present paper and two companion papers of Bender et $\mathrm{al}^{17}$ and Drake et $\mathrm{al}^{18}$ ) develops general criteria for the influence of surface steps on transition using a unique experimental facility. The experimental project was conducted in a Towing Wind Tunnel (for more details see the companion papers ${ }^{17,18}$ ) where a test model can be towed (moved) in still air allowing low background disturbance flow over the model. Models were designed to cover wide range of pressure gradients and the maximum transition Reynolds number reached on the models was more than $4 \times 10^{6}$.

The focus of the present paper is the experimental hot-wire anemometry investigations of the boundary layer flow on two models and a comparison with skin friction measurements obtained using arrays of Preston probes ${ }^{17}$. The goals of these measurements were: to document transition onset for a moving model in open air with natural disturbance environment ("flight" conditions) rather than for a static model in a conventional wind tunnel with an artificial disturbance environment; to find the mechanism of the transition in varied flow conditions such as stream-wise pressure gradient and size of excrescences; to compare the results on transition location obtained from velocity fluctuations and spectra measurements with those obtained from skin friction measurements. Another practical task was to document the background disturbance level.

\section{I.B. Dimensional considerations}

The excrescence geometries are defined in terms of their height, $\mathrm{k}$. The location of the step is described by the surface distance, $\mathrm{s}$, which is measured along the surface contour from the stagnation point to the location of the step. The nondimensional excrescence height is expressed as an excrescence Reynolds number, $\mathrm{Re}_{\mathrm{k}}$ :

$$
\mathrm{Re}_{\mathrm{k}}=\mathrm{u}_{\mathrm{k}} \mathrm{k} / v_{\mathrm{k}}
$$

This nondimensionalization is common in the literature and the parameters can be easily obtained from the measurement of the boundary layer velocity profile in an experimental situation, or by the use of simple two-dimensional boundary layer computational methods.

The nondimensionalized locations of the excrescence and transition can be expressed as Reynolds numbers, $\mathrm{Re}_{\mathrm{xk}}$ and $\mathrm{Re}_{\mathrm{xtr}}$, formed by integrating the boundary layer edge flow properties from the stagnation point along the surface, e.g.:

$$
\mathrm{Re}_{\mathrm{xk}}=\int(\mathrm{U} / v) \mathrm{ds}
$$

where integration is performed from leading edge to excrescence location for $\mathrm{Re}_{\mathrm{xk}}$ or from leading edge to transition location for $\mathrm{Re}_{\mathrm{xtr}}$ and $\mathrm{U}$ and $v$ are the velocity and kinematic viscosity in the freestream, at the boundary layer edge. Using $\mathrm{Re}_{\mathrm{xk}}$ to describe the location of the excrescences allows those flow properties that directly affect the development of the boundary layer to be taken into account. With a known pressure distribution, the parameters required to determine $\mathrm{Re}_{\mathrm{xk}}$ are easily obtained. 


\section{I.C. Background and previous work}

The literature on this topic is broad and a brief review of mostly recent work is given below. The experimental work of Wang \& Gaster $^{3}$ has addressed the effect of forward- and aft-facing step changes in surface height on the transition location in zero-pressure gradient (Blasius) boundary layers. By changing the unit Reynolds number via the freestream velocity and the roughness height while maintaining a constant streamwise step location they achieved parameter ranges of $\mathrm{x}_{\mathrm{k}} / \mathrm{k}<150$ and $1000<\mathrm{Re}_{\delta^{*}, \mathrm{k}}<1400$, approximately (where $\delta^{*}{ }_{\mathrm{x}, \mathrm{k}}$ is the displacement thickness of the unperturbed boundary layer at the step location), with $\operatorname{Re}_{\mathrm{xtr}}<2.4 \times 10^{6}$. The transition Reynolds number was determined using the streamwise location at which spikes indicative of nonlinear breakdown were first observed in the signal from a hotwire placed at the outer edge of the boundary layer. The data demonstrated good collapse to single curves of the change in $\mathrm{N}$-factor method ${ }^{4,5}, \Delta \mathrm{N}$, associated with forward- and aft-facing steps. Backward-facing steps led to larger reductions in transition Reynolds number for a given non-dimensional step height.

Crouch, Kosorygin \& $\mathrm{Ng}^{6}$ performed an experimental study of the influence of forward- and aft-facing steps at two streamwise locations corresponding to a favorable and adverse pressure gradient in a prescribed, complex pressure distribution. No consistent trends in the variation of transition Reynolds numbers between the different types of pressure gradient can be determined, but the two general curves for the $\mathrm{N}$-factor corrections introduced by forward- and aft-facing steps as a function of $\mathrm{k} / \delta^{*}$ indicate that the favorable pressure gradient leads to more abrupt changes in $\Delta \mathrm{N}$ bounded by the same approximate curve as for the adverse pressure gradient. The computational work of Nayfeh ${ }^{7}$ determined the pressure distribution associated with steps and humps and the corresponding stability analysis indicated regions of strong stabilization associated with even short lengths of decreasing pressure.

Perraud \& Seraudie ${ }^{8}$ made experimental and computational determinations of the critical Reynolds numbers and changes to $\Delta \mathrm{N}$ for Blasius and airfoil boundary layers, while Tani ${ }^{9}$ summarized work on twodimensional cylindrical roughness elements, again obtaining single curves for a given disturbance environment. He noted that the influence of background turbulence was confined to the low nondimensional step heights, corresponding to a lower undisturbed transition Reynolds number.

The influence of the different transition indicators, namely the onset of turbulent spikes versus pressure measurements such as Preston probe indicators of a minimum in dynamic pressure followed by a rise in wall shear stress on the empirical scaling results has not been explicitly detailed.

In this work, part of a larger program on the prediction of manufacturing tolerances for laminar flow, we investigate the scaling of the transition location in an experimental, incompressible boundary layer subjected to disturbance by two-dimensional surface excrescences at varying Reynolds numbers based on displacement thickness and pressure gradients of varying strength. The extended empirical relationships between transition location and excrescence parameters are of interest rather than simply a critical Reynolds number, because of the application to finite extent laminar flow airfoils.

\section{Approach and instrumentation}

The experiments were performed in the Towing Wind Tunnel facility ${ }^{10}$, which is owned and operated by Tohoku University. The facility is located in Hyuga, Miyazaki Prefecture, Japan. The facility consists of: a carrier vehicle called the "HART vehicle" on which a test model was placed, a guideway track in which the HART vehicle runs (the name "HART" comes from Hyuga Aerodynamic Research facility by Towing, the facility is located near the town of Hyuga), and a control system. Two models were used to produce different pressure gradients (figure 1). The two dimensional step was created by moving a leading edge assembly piece (19 inches long) with respect to the rest of the model. The step could be set as aft or forward facing with adjustable height. The models were mounted vertically on the HART carrier vehicle between two splitter plates (figure 2). For more details about the test facility and model design refer to Bender et $\mathrm{al}^{17}$.

The objective of the model aerodynamic design was to create models that preserved near-constant chordwise and spanwise acceleration parameter:

$$
\mathrm{K}=\mathrm{v} / \mathrm{U}^{2}{ }_{\mathrm{e}}\left(\mathrm{dU}_{\mathrm{e}} / \mathrm{dx}\right) .
$$


The first model, with nominally zero pressure gradient, corresponded to an actual $\mathrm{K}=0.2 \times 10^{-7}$, denoted $\mathrm{K}_{0}$ ("Gradient-Zero" model). The second model ("Gradient A") had $\mathrm{K}_{\mathrm{A}}=0.5 \times 10^{-7}$.

Multiple measurement methods were used in order to provide redundancy, comparison and confidence, as well as to add to the physical foundation of the results. The parameters measured during the test included: pressure coefficient distributions, boundary layer transition location, skin friction coefficients, boundary layer velocity profiles and disturbance frequency distributions. The static pressure taps were used to measure the pressure coefficient distributions on the models. A boundary layer traverse was used to measure boundary layer velocity profiles at the step location in order to reduce step heights to dimensionless $\mathrm{Re}_{\mathrm{k}}$ values. Preston tubes were used to measure skin friction distributions. Each Preston tube had a corresponding static tap located at the same chordwise location. The resulting pressure differences were converted to skin friction coefficients using Patel's calibration ${ }^{11}$ for turbulent boundary layers and Poll's calibration ${ }^{12}$ for laminar boundary layers. The transition location was determined to correspond to the position at which the spatially-extrapolated skin friction exceeded the measured clean plate laminar reference at that location by $30 \%$. More details about skin friction and static pressure measurements and corresponding instrumentation can be found in Bender et $\mathrm{al}^{17}$.

Hot wire anemometry was used to obtain velocity spectra for each model. In addition the two approaches, Preston tubes and hot-wires, could be compared to validate the results of the transition location measurements. A standard four-channel constant temperature hot-wire anemometer (AA Lab System AN1005) was used with four hot-wire boundary-layer probes (Dantec). The velocity signals from the anemometer amplifiers were acquired using a National Instruments Data Acquisition card and saved to an onboard laptop computer. The hot-wire holders were attached to the surface with specially-designed mounting holders at a nominally constant height of 0.02 ", or $y / \delta^{*} \sim \mathrm{O}(1)$. The distance between hot-wire sensor and surface was measured from the images obtained with a Nikon D300 digital camera fitted with a Tamron SPAF $180 \mathrm{~mm}$ Lens, with accuracy 50-100 microns. Calibration of hot-wires was performed using a home-made calibrator unit versus known flow velocity measured simultaneously with a Pitot tube. Model vibrations were recorded with two accelerometers (Omega). Vibrations could be considered as a source of flow fluctuations and need to be monitored and controlled, if necessary. A low disturbance environment with a conservative estimate of turbulence intensity $<0.05-0.10 \%$ was recorded for all runs, once the common mode electronic noise correlated between hot-wires had been removed.

The 2-D boundary layer code IBL was used to provide both reference laminar and turbulent skin friction coefficient distributions on the models for a given pressure distribution, and boundary layer parameters such as the displacement thickness, momentum thickness, boundary layer thickness, and velocity profiles, as a function of $\mathrm{x}$. The laminar boundary layer computations were used as input to the Rapid $N \mathrm{~N}$-factor analysis code ${ }^{13}$ in order to perform a preliminary investigation of the stability of the boundary layer under each pressure gradient. This analysis uses Pad'e approximants and a parallel flow assumption to obtain a functional form for the neutral stability loop and thus permits rapid, accurate estimation of the eigenvalues to determine instability amplification rates across a range of frequencies. Errors are introduced because of the assumptions associated with fitting local Falkner-Skan profiles to the actual flow and the neglect of the streamwise gradient terms, but under some circumstances results representative of the experimental conditions were obtained.

\section{Results}

Detailed characterization of the flow properties was performed for both models. The experimentally obtained distributions of the pressure coefficients and acceleration parameters are shown in figures 3 and 4 respectively. There was a significant acceleration and deceleration near the leading edge especially for zero-pressure-gradient model (figure 4), which could lead to the region of enhanced receptivity there and likely makes the transition mechanism more complicated than the simple Rapid $N$ analysis allows. In order to perform stability analysis the variation of shape factor, $\mathrm{H}$, the ratio of displacement thickness to momentum thickness, and the pressure parameter, $\beta$, the measure of the pressure gradient for Falkner-Skan wedge flows ${ }^{19}$ is required for our models. The dependence of the shape factors calculated as function of stream-wise distance (figure 5) indicates that the flows for both models were not of Falkner-Skan types. In order to find the variation of $\beta$, a local Falkner-Skan approximation was used, i.e. we consider the shapes of our models as sets of very small wedges. Each such wedge has its own inclination angle and the flow over each wedge has its own shape factor. Using unique relation between $H$ and $\beta$ for similar flows ${ }^{14}$ we can determine $\beta$ for all stream-wise positions (figure 6). 
Figures 7 through 10 show the relationships between transition and roughness Reynolds numbers for both models as determined from the hot-wire measurements. The qualitative criteria for the transition from hot-wire measurements were a fully intermittent velocity history and broad power spectrum at the probe location, nominally $\mathrm{y} \sim \delta^{*}$. The low spatial resolution in the hot-wire measurements did not usually permit determination of the exact location of the transition point (the transition location was found not better than somewhere between two successive hot-wires corresponding to two symbols connected by line in the plots). The transition locations inferred from the hot-wire measurements were compared with those found from Preston tube measurements, presented over a range of non-dimensional excrescence location Reynolds number, $\mathrm{Re}_{\mathrm{sk}}$. The two different methods gave consistent results and both showed that the aftfacing step caused a stronger reduction in transition Reynolds number than a forward-facing step of the same non-dimensional magnitude. In the limit of the very large step height $\operatorname{Re}_{\mathrm{k}}$ the transition location for both types of steps was indistinguishable. The stronger stabilizing effect of the higher pressure gradient can be clearly seen if a comparison is made, for example, of the aft-facing step cases (figures 7 and 9): doubling the acceleration parameter leads to an approximate doubling of the step height which is required to trigger transition in the high transition Reynolds number limit.

The results for the transition Reynolds number as function of non-dimensional step height, $\mathrm{k} / \delta^{*}$, obtained from different methods are shown in figures 11-14. The data extracted from Wang \& $\mathrm{Gaster}^{3}$ for the case of flat plate $(\mathrm{K}=0)$ in a low-turbulence wind tunnel (turbulence intensity less than $0.01 \%$ ) are compared with our zero pressure gradient model in figures 11-12. Wang \& Gaster identified the transition location when the intermittency spikes first appeared in their velocity signal at the outer edge of the boundary layer. In our case the transition Reynolds numbers are lower by factor of 2 or more for the same non-dimensional step height, at least for the forward-facing step. The reason for this may be due to higher level of free stream turbulence on our models. Figures 13-14 show the results for the favorable pressure gradient model. As in the previous plots, the stronger reduction in the transition Reynolds number can be observed for lower pressure gradient and aft-facing step.

The hot-wire measurements of the velocity power spectrum evolution with stream-wise distance for the zero pressure gradient model are shown in figure 15. These sample measurements were performed at $\mathrm{U}=17.3 \mathrm{~m} / \mathrm{s}$ and with hot wires distributed from just upstream of the step to close to the transition point indicated by the skin friction results. The spectra indicated the presence of the peaks between $200 \mathrm{~Hz}$ and $300 \mathrm{~Hz}$. As the successive stability analysis showed, the peaks corresponded to unstable TollmienSchlichting (T-S) waves (see below). T-S waves were also observed for the case of a clean plate (without step) but the transition was not observed on the plate (the critical Reynolds was not reached for this speed). Nevertheless, the zero step case served as a reference to compare with simulations. The example of the comparison of the Rapid $\mathrm{N}$-factor computations with the experimentally found $\mathrm{N}$-factors for the GradientZero model without step is shown in figure 16 . The example corresponds to $U=12.7 \mathrm{~m} / \mathrm{s}$. The lines in the plots show $\mathrm{N}$-factors computed for individual frequencies (incremented by $17 \mathrm{~Hz}$ in the range $66-418 \mathrm{~Hz}$ ) as functions of stream-wise position. The envelope to the lines gives the maximal $\mathrm{N}$-factor. The symbols are the experimental $\mathrm{N}$-factors determined from the velocity power spectra. It can be seen that the experimental data are in the right range of stream-wise locations where the maximal amplification is observed and the corresponding experimental frequencies are close to computed ones. The maximally amplified frequencies from the stability analysis $(167 \mathrm{~Hz}$ and $150 \mathrm{~Hz})$ are close to the frequencies from the experiment $(153 \mathrm{~Hz}$ and $144 \mathrm{~Hz}$ ) for the same stream-wise locations. Two different ways of calculating of the amplification were used. The open symbols in the plots correspond to the case when the level of noise for the first upstream hot-wire was used as the initial power of T-S waves (note that this level is likely contaminated by common mode electronic noise). The values of the $\mathrm{N}$-factors calculated in this way were smaller than the computed ones. The filled symbols correspond to the case when the level of noise for a zero flow in calm environment (data not shown) was taken as the initial power. The $\mathrm{N}$-factors in this case showed much closer agreement to the simulations. The presence of steps did not alter the T-S wave frequency as was observed from the spectra frequency data of T-S waves for different steps, but did increase the amplitudes of the T-S disturbances.

The stability diagram showing which frequencies are unstable for a given Reynolds number is presented in figure 17. The line in the plot is the neutral stability curve taken from simulations of Wazzan ${ }^{16}$ for parallel similar flow with stream-wise pressure gradient. The symbols in the plot are our experimental data for the case of the zero pressure gradient model without a step. The two different values of $\beta=0.052$ and 0.073 are for two different stream-wise positions s=27in and 32in correspondingly. It can be seen that the points are well inside of the unstable region corresponding to unstable T-S waves. It was concluded that 
the transition process for the zero pressure gradient model was linear through the generation of unstable T$\mathrm{S}$ waves. For the favorable pressure gradient model, the minimal critical Reynolds number for T-S waves using the local Falkner-Skan approximation was not reached on the model.

An example of the skin friction measurements for different $U$ conditions is shown in figure 18 for the case of the zero pressure gradient model with a forward facing step. Figure 19 shows velocity RMS (rootmean-square) distributions for different $\mathrm{U}$. It can be seen that the transition onset estimated from the velocity data occurred a little earlier than that determined from the $\mathrm{Cf}$ distributions. This can be explained by the fact that the velocity spectral broadening and the degree of intermittency (and RMS) are sensitive to the perturbations to the mean velocity profile, whereas the $\mathrm{Cf}$ increases when the boundary layer velocity profile changes from laminar to turbulent. The latter is confirmed in the plot shown in figure 20: an increase in $\mathrm{Cf}$ was a response to a change in the velocity from laminar towards a turbulent profile.

\section{Summary}

Hot-wire anemometry was used to document the background disturbance level and investigate disturbance growth in the study of the effect of a favorable pressure gradient on the influence of surface steps on boundary layer transition performed in a towing facility. The stabilizing effect of the pressure gradient can be clearly seen in both hot-wire velocity data and skin friction measurements reported in more details in a companion paper. The measurements confirmed that aft-facing steps lead to more serious perturbations to the laminar boundary layer and earlier transition than forward-facing steps. The excrescence relationships showed a significant difference from earlier measurements in a different disturbance environment and with slightly different step geometries.

This paper is a companion paper to two other papers ${ }^{17,18}$, which describe the approach and the objective of the study, details of the experimental technique, the final excrescence relation results developed in the study, and the application of those results to laminar flow manufacturing tolerance guidelines.

\section{Acknowledgments}

The authors acknowledge funding and support from the Air Force Research Laboratory, Prof. Mike Gaster of Queen Mary College, University of London, for the use of the Rapid-N code, and Prof. Bill Saric for useful advice.

\section{References}

${ }^{1}$ Drake, Bender, Solomon, and Vavra, "Air Vehicle Technology Integration Program (AVTIP) Delivery Order 0020: Prediction of Manufacturing Tolerances for Laminar Flow Final Report," AFRL-VA-WP-TR-2005-3060, June 2005.

${ }^{2}$ Bender, and Drake, “Air Vehicle Technology Integration Program (AVTIP) Delivery Order 0020: Prediction of Manufacturing Tolerances for Laminar Flow Task 6 Final Report," AFRL-VA-WP-TR-2007-3086, September 2006.

${ }^{3}$ Wang Y.U. and Gaster M. (2005), "Effect of surface steps on boundary layer transition”, Exp. In Fluids, 39, pp. 679-686.

${ }^{4}$ Van Ingen J.L. (1956) "A suggested semi-empirical method for the calculation of the boundary layer transition region", Department of Aeronautics Engineering, Deft Institute of Technology,

Delft, The Netherlands, report VTH-74.

${ }^{5}$ Smith A.M.O., Gamberoni N. (1956) "Transition, pressure gradient and stability theory", Douglas Aircraft Co., technical report no ES-26388.

${ }^{6}$ Crouch, J. D., Kosorygin, V. S. and Ng, L. L. (2006) "Modeling the effects of steps on boundary-layer transition," in IUTAM Symposium on Laminar-Turbulent Transition, ed. Govindarajan, R., pp 37-44, Fluid Mechanics and its Applications (vol. 78), Springer Netherlands.

${ }^{7}$ Nayfeh, A.H. (1992) "Influence of two-dimensional imperfections on laminar flow" SAE Paper No. 921990.

${ }^{8}$ Perraud, J. and Seraudie, A. (2000) "Effects of steps and gaps on 2D and 3D transition" ECCOMAS 2000.

${ }^{9}$ Tani, I. (1969) "Boundary-layer transition” Annu. Rev. Fluid Mech., 1, pp. 169-196

${ }^{10}$ Yoshioka, Kikuchi, Ohta, Kato, Song, and Kohama, "Measurement of ground effect and boundary-layer transition by towing wind tunnel," 2009 Fluid Dyn. Res. 41021408 (12pp). 
${ }^{11}$ Patel, V.C., "Calibration of the Preston Tube and Limitations on its Use in Pressure Gradients", Engineering Laboratory, Cambridge University, December 21, 1964.

${ }^{12}$ Poll, P.I.A., "A Note on the Use of Surface Pitot Tubes for the Measurement of Skin Friction in Laminar Boundary Layers", College of Aeronautics Report 8307, Cranfield Institute of Technology, Cranfield, Bedford, U.K., March 1983.

${ }^{13}$ Gaster M. and Jiang F. (1994), "A rapid scheme for estimating transition on wings by linear stability theory", Proc. $19^{\text {th }}$ Congress of ICAS, Vol. 2, pp. 1104-1113, Anaheim, CA.

${ }^{14}$ Obremski H.J., et al. (1969), "A portfolio of stability characteristics of incompressible boundary layers", AGARDograph No.134, NATO, Paris.

${ }^{15}$ Mack L.M. (1977) "Transition prediction and linear stability theory", AGARD pp. 1-1-1-22.

${ }^{16}$ Wazzan A.R., Gazley C., and Smith A.M.O. (1968), "Spatial and temporal stability charts for the Falkner-Skan boundary layer profiles", Douglas aircraft Co. Report DAC-67087.

${ }^{17}$ Bender A.M., Elliott J.R., Shinagawa Y., Korntheuer A., Drake A., Westphal R.V., Gerashchenko S., McKeon B.J., and Yoshioka S. (2010), "An Approach to Measuring Step Excrescence Effects in the Presence of a Pressure Gradient”, Paper 2010-373, 48 ${ }^{\text {th }}$ AIAA Aerospace Sciences Meeting.

${ }^{18}$ Drake A., Bender A.M., Korntheuer A., Westphal R.V., McKeon B.J., Gerashchenko S., Rohe W., and Dale G. (2010), "Step Excrescence Effects for Manufacturing Tolerances on Laminar Flow Wings", Paper 2010-375, 48 AIAA Aerospace Sciences Meeting.

${ }^{19}$ White F.M. (2006), "Viscous fluid flow", McGraw-Hill International Edition. 


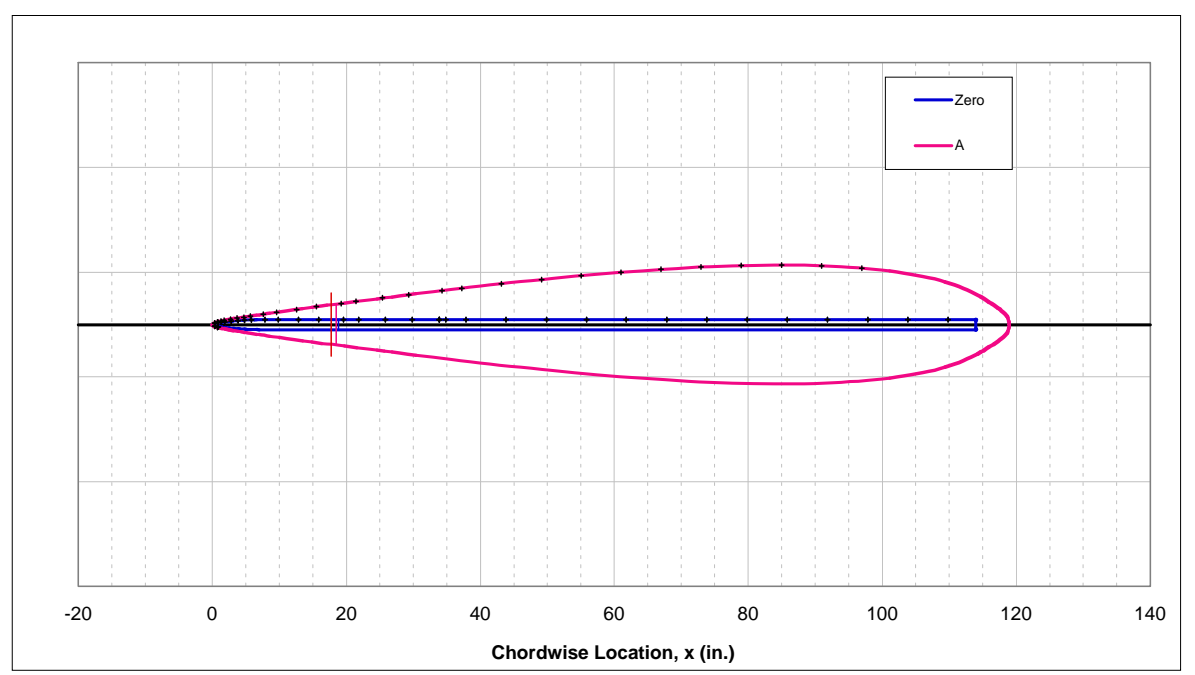

Figure 1. Schematics of the model geometries.

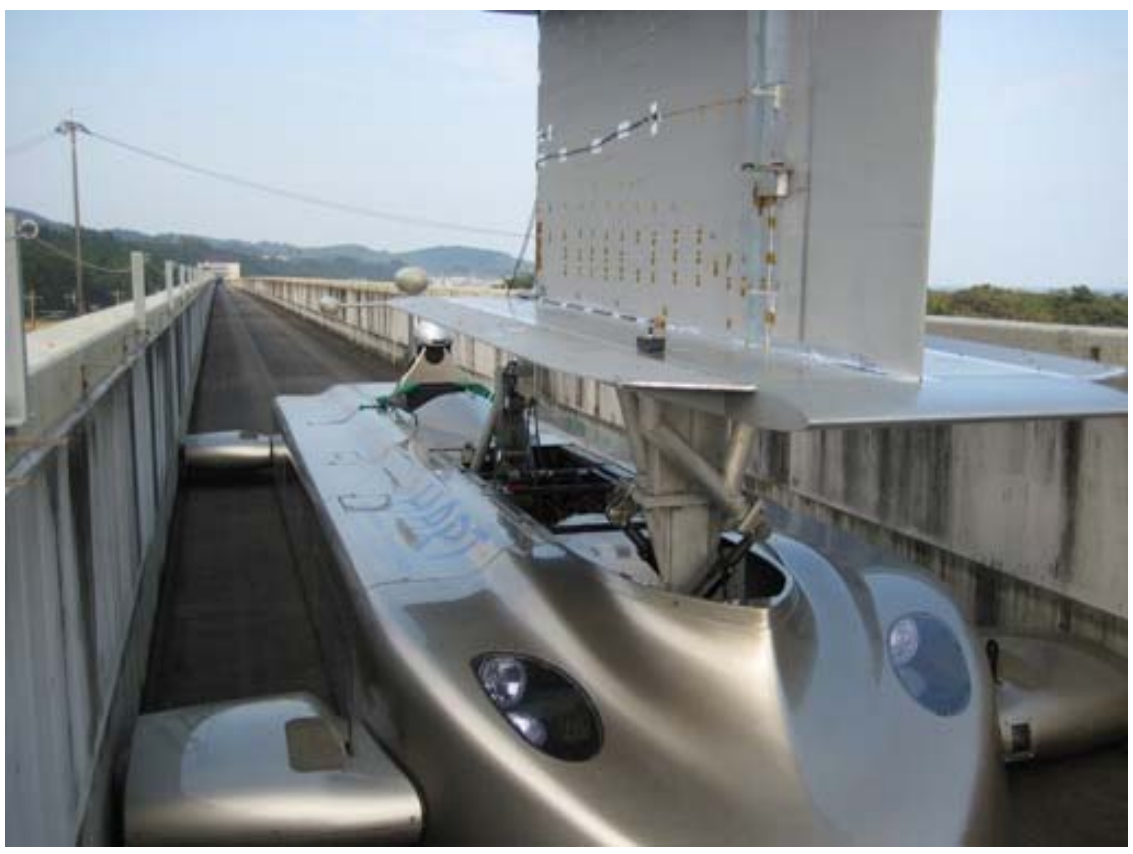

Figure 2. HART vehicle with zero pressure gradient model installed. 


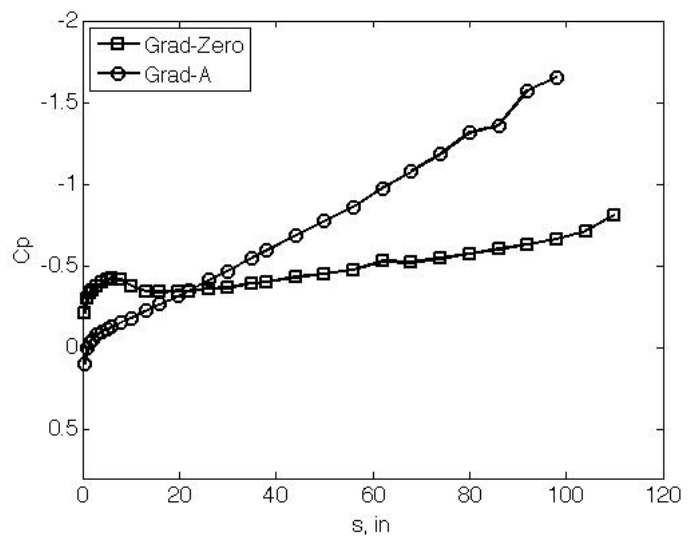

Figure 3. Distributions of pressure coefficients (at $U=30 \mathrm{~m} / \mathrm{s}$ ) for both models.

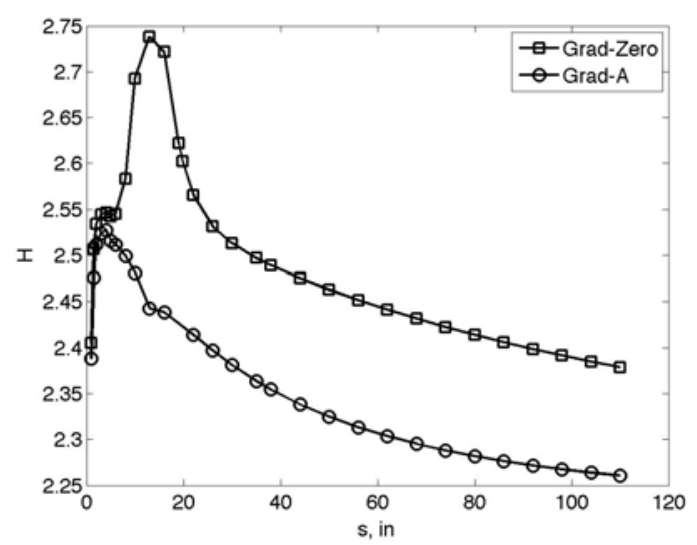

Figure 5. Distributions of shape factors (at $\mathrm{U}=22.3 \mathrm{~m} / \mathrm{s}$ for zero pressure gradient model and $\mathrm{U}=\mathbf{2 4} \mathrm{m} / \mathrm{s}$ for favorable pressure gradient).

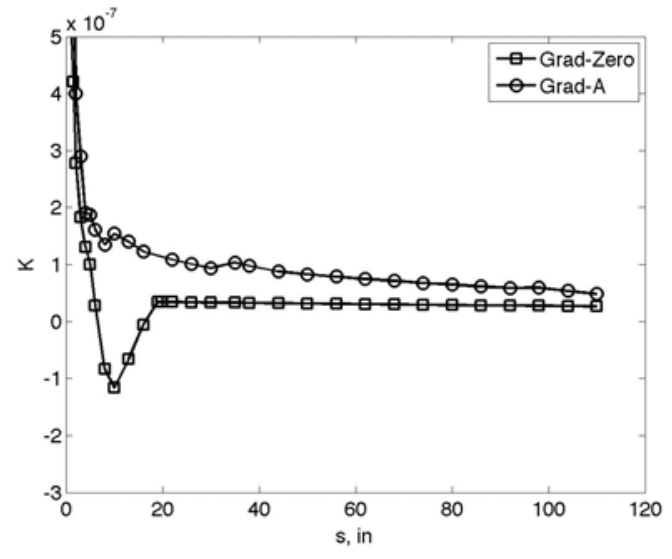

Figure 4. Distributions of acceleration parameters (at $U=22.3 \mathrm{~m} / \mathrm{s}$ for zero pressure gradient model and $U=24 \mathrm{~m} / \mathrm{s}$ for favorable pressure gradient).

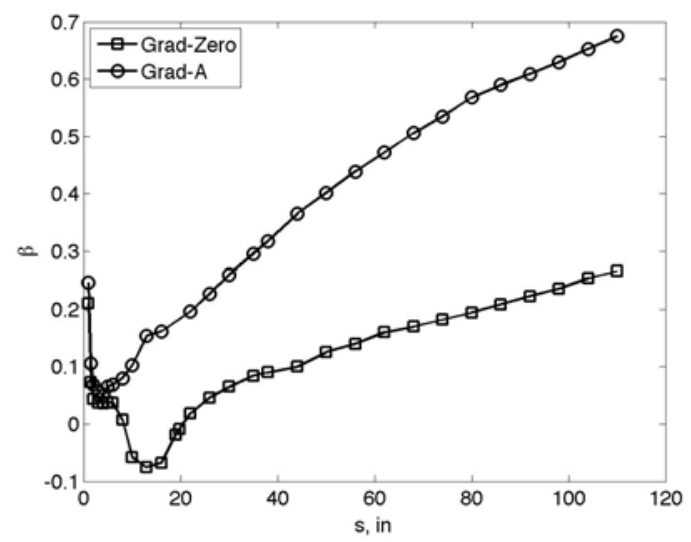

Figure 6. Distributions of pressure parameters using the local Falkner-Skan

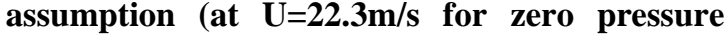
gradient model and $U=24 \mathrm{~m} / \mathrm{s}$ for favorable pressure gradient). 


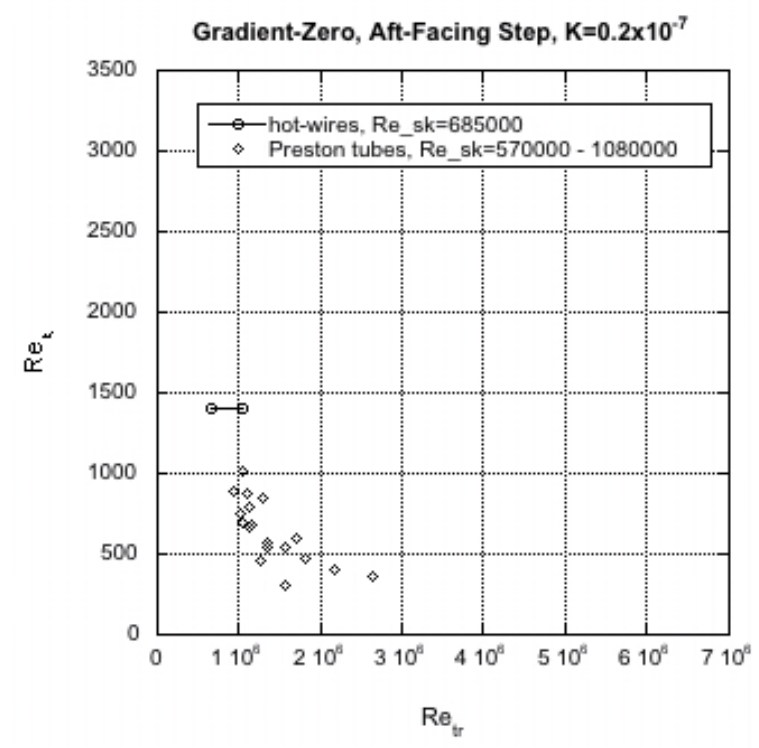

Figure 7. Transition location for aft-facing steps on zero pressure gradient model.

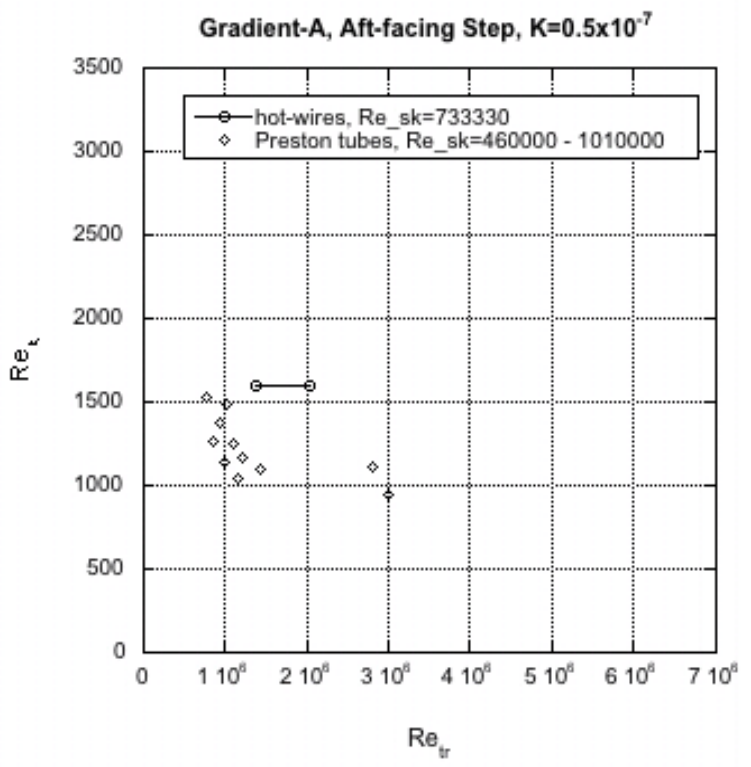

Figure 9. Transition location for aft-facing steps on favorable pressure gradient model.

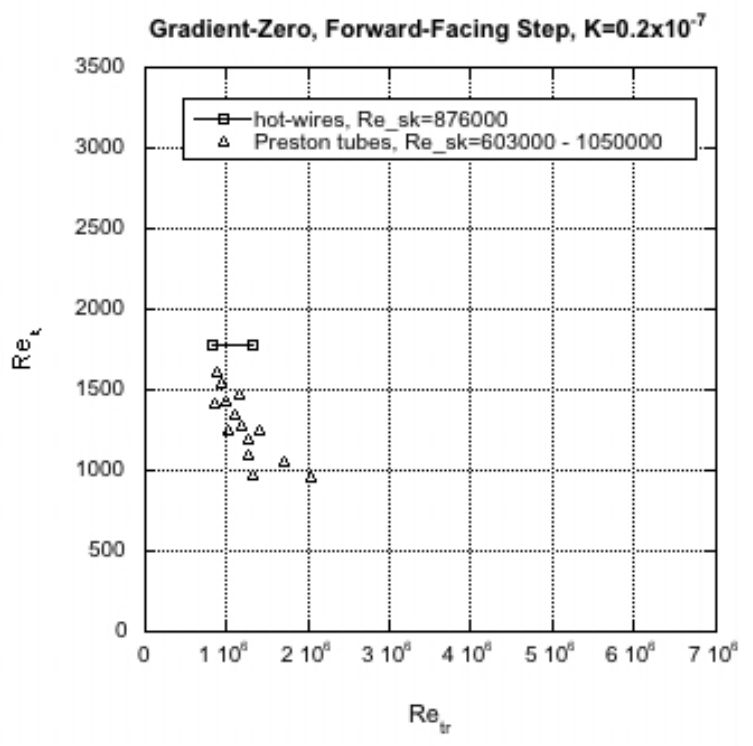

Figure 8. Transition location for forwardfacing steps on zero pressure gradient model.

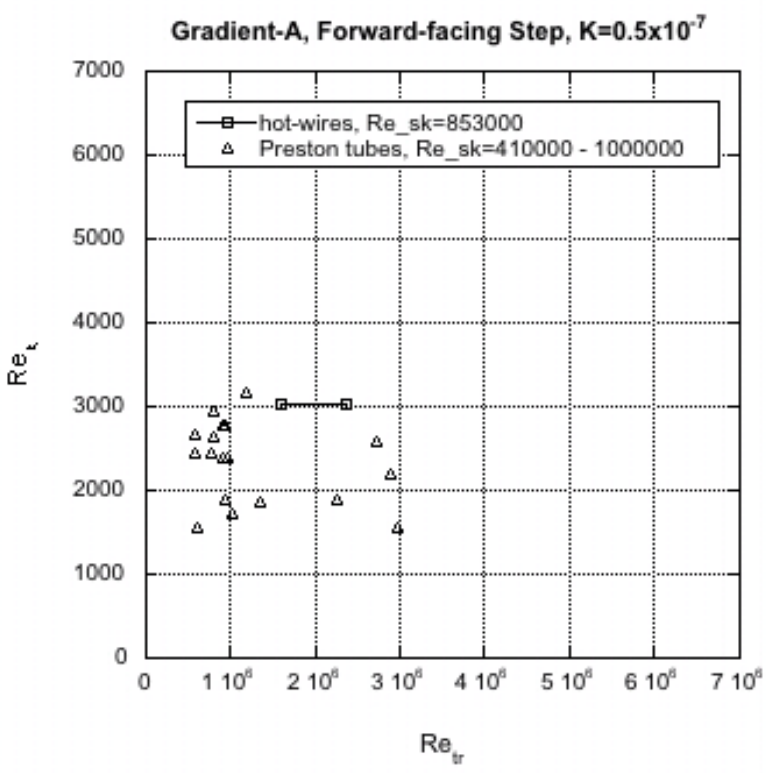

Figure 10. Transition location for forwardfacing steps on favorable pressure gradient model. 


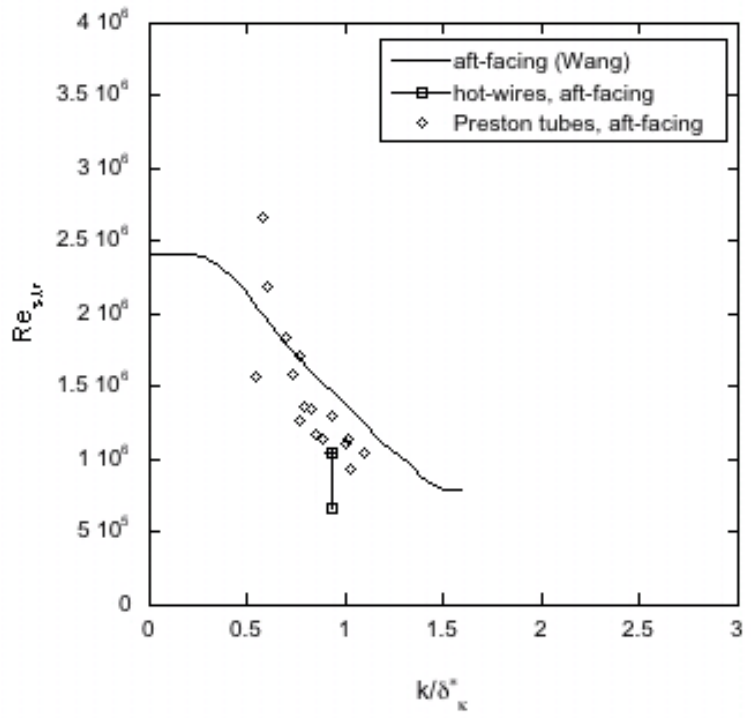

Figure 11. Transition location as function of aft-facing step height from different methods (solid line - flat plate data from Wang \& Gaster, symbols -zero pressure gradient model).

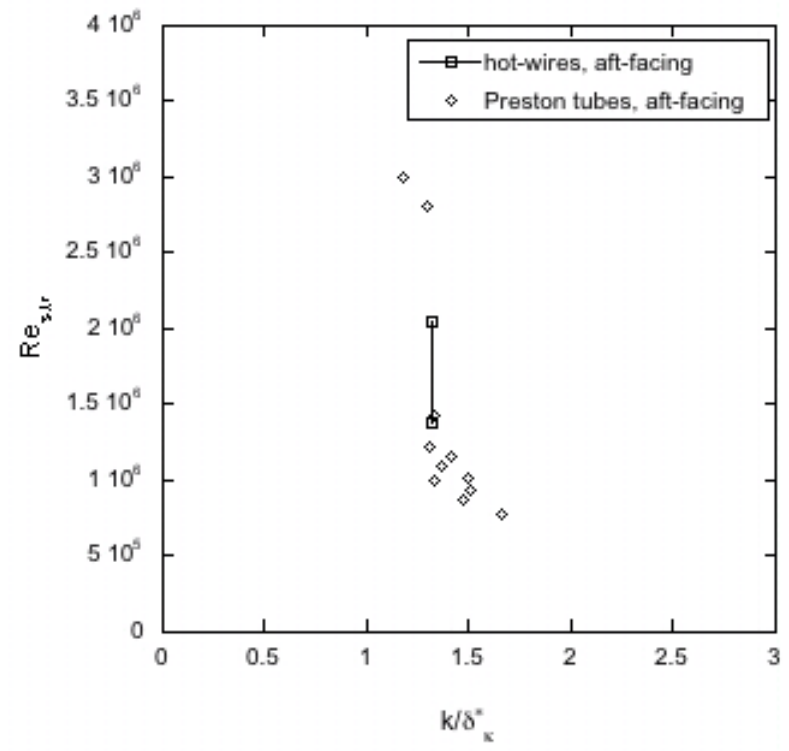

Figure 13. Transition location as function of aft-facing step height from different methods (favorable pressure gradient model).

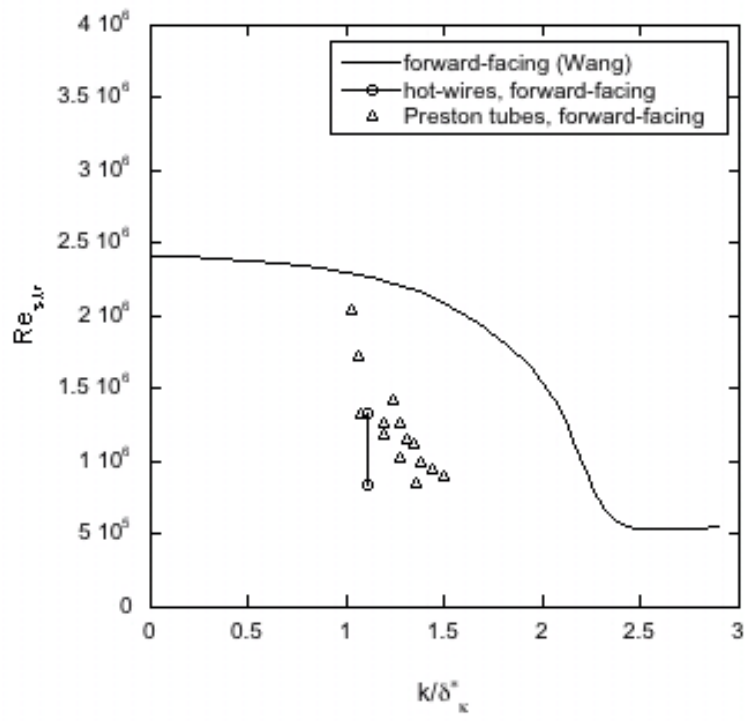

Figure 12. Transition location as function of forward-facing step height from different methods (solid line - flat plate data from Wang \& Gaster, symbols - zero pressure gradient model).

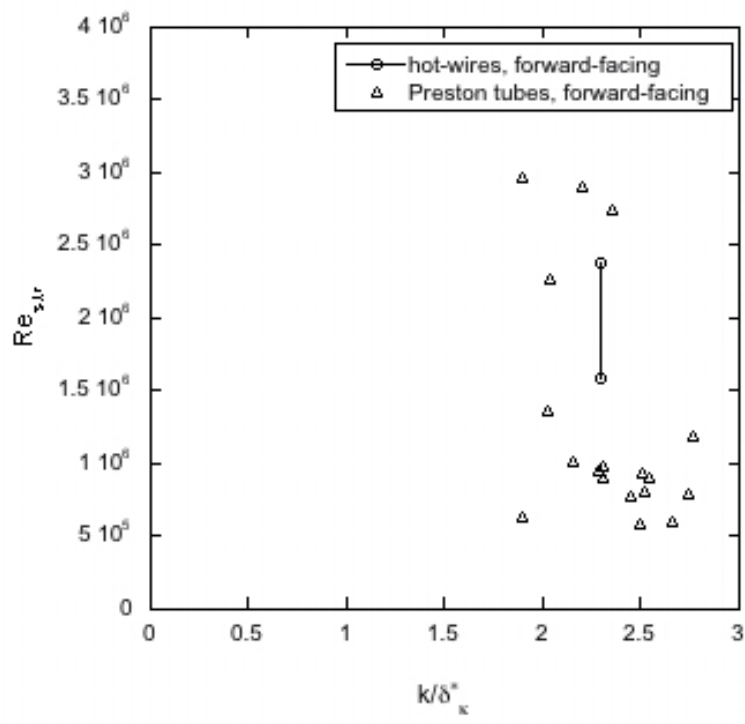

Figure 14. Transition location as function of forward-facing step height from different methods (favorable pressure gradient model). 


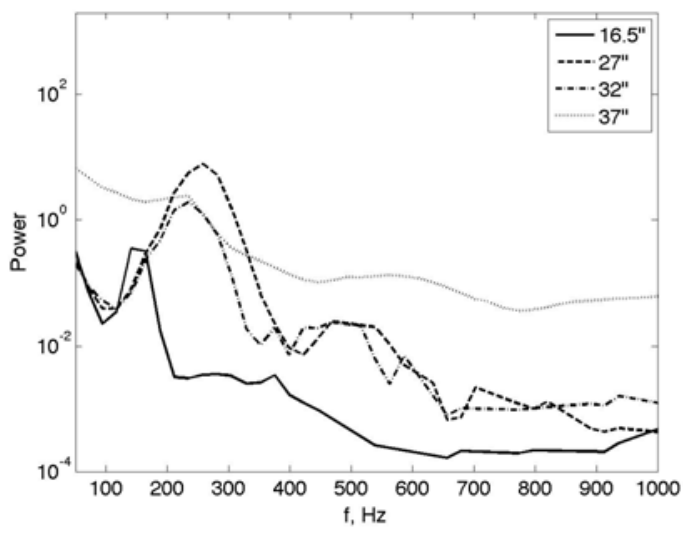

Figure 15. Example of the velocity power spectra evolution with stream-wise distance for the zero pressure gradient model. T-S waves were observed at about $260 \mathrm{~Hz}$ at $s=27-32 \mathrm{in}$. Step height $\mathrm{k} / \delta^{*}=0.93, \operatorname{Re}_{\mathrm{s}, \mathrm{k}}=685000$.

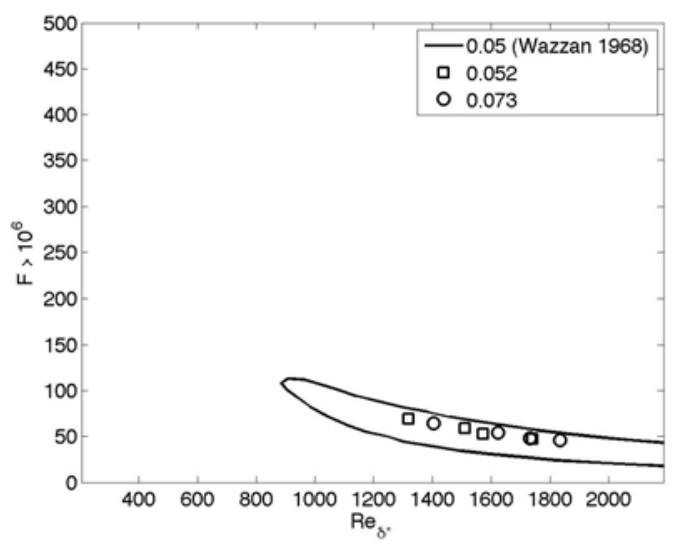

Figure 17. Stability diagram. Solid line results from simulations of Wazzan for pressure gradient $\beta=0.05$, symbols - unstable frequencies observed for zero pressure gradient model with zero step height for two pressure gradients $\beta=0.052$ (squares) and $\beta=0.073$ (circles).

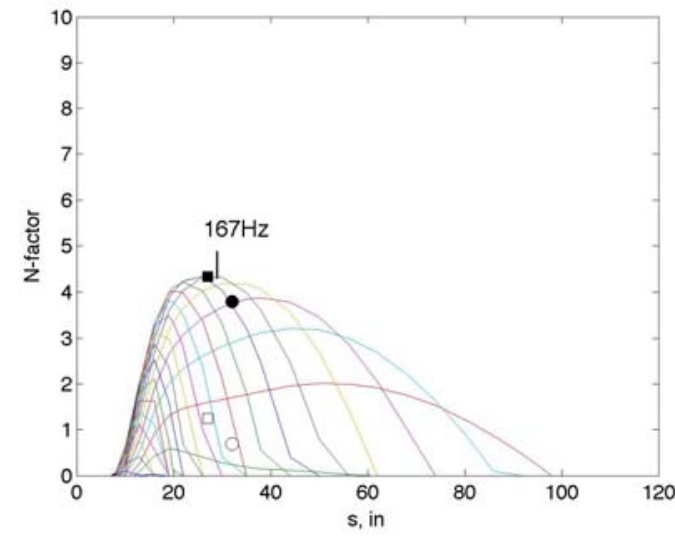

Figure 16. Comparison of $\mathrm{N}$-factors from Rapid-N calculation (lines) and from the experiments (symbols). Open and filled symbols correspond to different choice of the initial amplitude of T-S wave (see text).

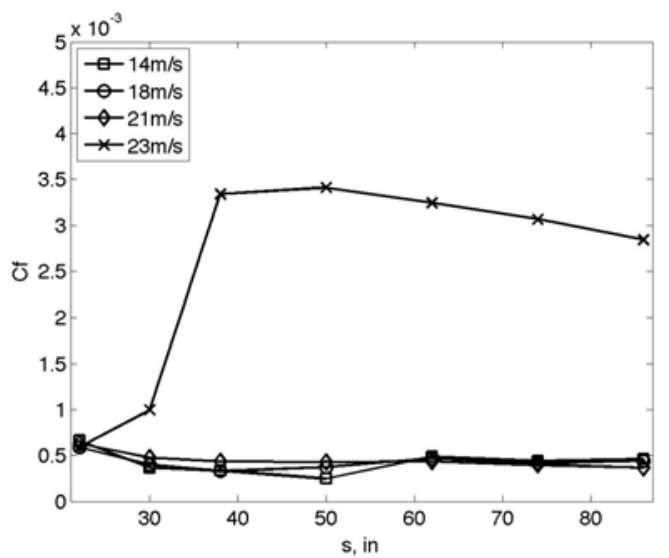

Figure 18. Skin friction distributions for different free stream speeds, U. Zero pressure gradient model with a forward facing step Transition was observed at $\mathrm{U}=\mathbf{2 3} \mathrm{m} / \mathrm{s}, \mathrm{s}=37 \mathrm{in}$ $\left(\operatorname{Re}_{\mathrm{s}, \mathrm{tr}}=1980000\right)$ and $\mathrm{Re}_{\mathrm{s}, \mathrm{k}}=876000$. Step height $\mathrm{k} / \delta^{*}=\mathbf{1 . 0 6}$. 


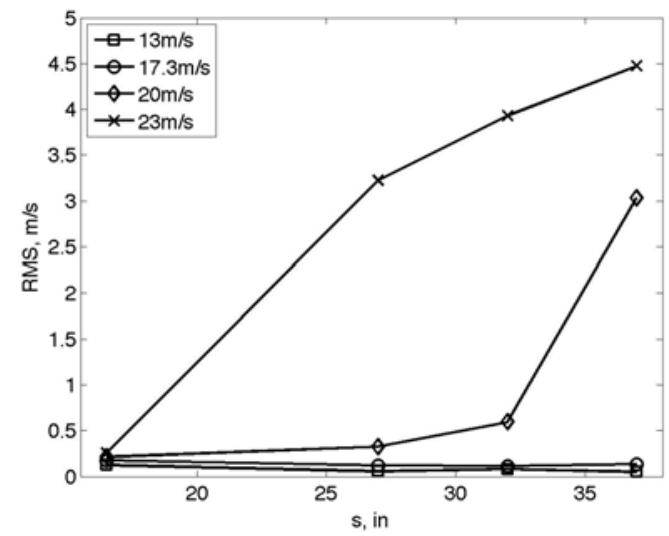

Figure 19. Velocity RMS distributions for different free stream speeds, U. Zero pressure gradient model with a forward facing step. Transition was observed at $U=23 \mathrm{~m} / \mathrm{s}, \mathrm{s}=26 \mathrm{in}$ $\left(\operatorname{Re}_{\mathrm{s}, \mathrm{tr}}=1300000\right)$ and $\operatorname{Re}_{\mathrm{s}, \mathrm{k}}=876000$ Step height $\mathrm{k} / \delta^{*}=\mathbf{1 . 0 6}$.

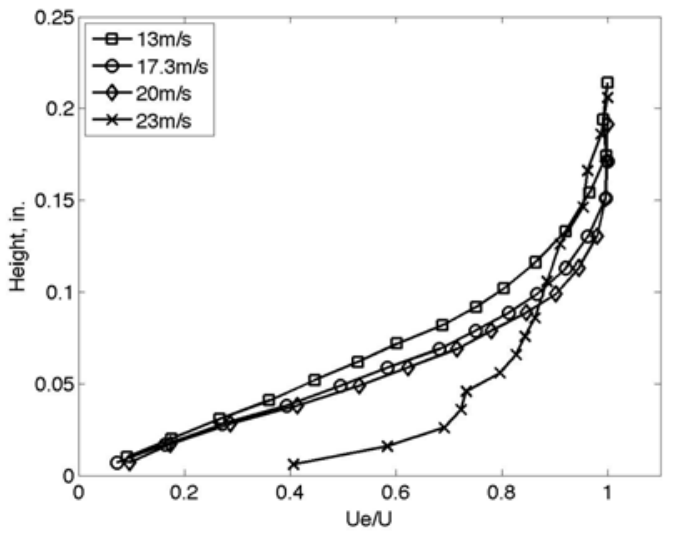

Figure 20. Boundary layer velocity profiles for different free stream speeds, $U$, and at the same stream-wise location $\mathrm{s}=37 \mathrm{in}$. Zero pressure gradient model with a forward facing step. Step height $k / \delta^{*}=1.06$. 